\title{
Research on Tool Wear Prediction Based on Deep Residual Network
}

\author{
Kai Tang ${ }^{1, a}$, Xiaodong Wang ${ }^{1, b}$, Wenzhi Hu${ }^{1, c}$, and Yuan Yang ${ }^{1, d}$ \\ ${ }^{1}$ Systems Engineering Research Institute, Beijing 100036, China. \\ awangzi20010@163.com, ’xdwxd@163.com, 'happy-fly-man@163.com, doubley2006@126.com
}

Keywords: Tool wear, convolutional neural network, residual network.

\begin{abstract}
The convolution neural network with deep residual network structure is applied to the prediction of milling tool wear, and the high prediction accuracy is obtained. Based on the data set of the 2010 PHM data competition, this paper constructs 64000 data samples from the total life cycle wear data of three milling tools. Then a 12-layer deep residual network is trained based on these data. Compared with the classical signal analysis method, this method is more convenient for modeling and application because no signal feature extraction is needed, and the generalization ability of the model is better.
\end{abstract}

\section{Introduction}

In 1998, the concept of a convolution neural network was proposed by LeCun [1]. The core idea of convolution neural network is to extract local features in input information by using filters, so as to achieve parameter sharing, and introduce pooling operation in neural network to reduce network parameters, which makes it easier for neural network to learn the characteristics of data. Since then, LeNet-5, AlexNet [2], VGG [3], GoogLeNet [4], ResNet [5] and other structural models have been developed in the process of neural network development. The structure of the convolution neural network becomes more complex, and the number of layers becomes deeper. Meanwhile, the recognition effect of the model is improved step by step. Due to the difficulty of data acquisition, the research and application of convolution neural network mostly focus on the areas of image recognition, voice recognition, video recognition and so on, while the research in the industrial field is far less intense.

In the related research of tool state monitoring, the researchers are puzzled by the problems of the prediction accuracy and generalization ability of the tool wear prediction model [6, 7]. This causes the model cannot be applied in actual processing in the short term. In this study, a deep residual convolution neural network algorithm is introduced in order to acquire a better generalization model and modeling method, thereby improving the practical value of the prediction model.

\section{Problem}

To acquire data about tool condition in machining process, a 3-component platform dynamometer made by Kistler was mounted between the workpiece and the fixture tools to measure cutting forces. Meanwhile the cutter's flank wear was measured after a machining step using a LEICA MZ12 microscopy system. Then all of the data were processed into a force signal fragment with length of 1024, and the tool wear degree corresponding to the force signal. We finally got 64000 sets of such data. The function of the convolution neural network model established in this study is to map a segment of force signal to a value between 0 and 1 . We use " $X$ " to indicate the cutting forces in $\mathrm{x}$ axis direction signal in the milling process, which is measured in one period of time, and the unit is Newton. "S" indicates the degree of tool wear, whose value is between 0 and 1 . S is 0 when the tool does not wear out while $\mathrm{S}$ is 1 when the tool can not be used again. $\mathrm{S}$ can be converted to the actual wear of the tool through a simple transformation. 


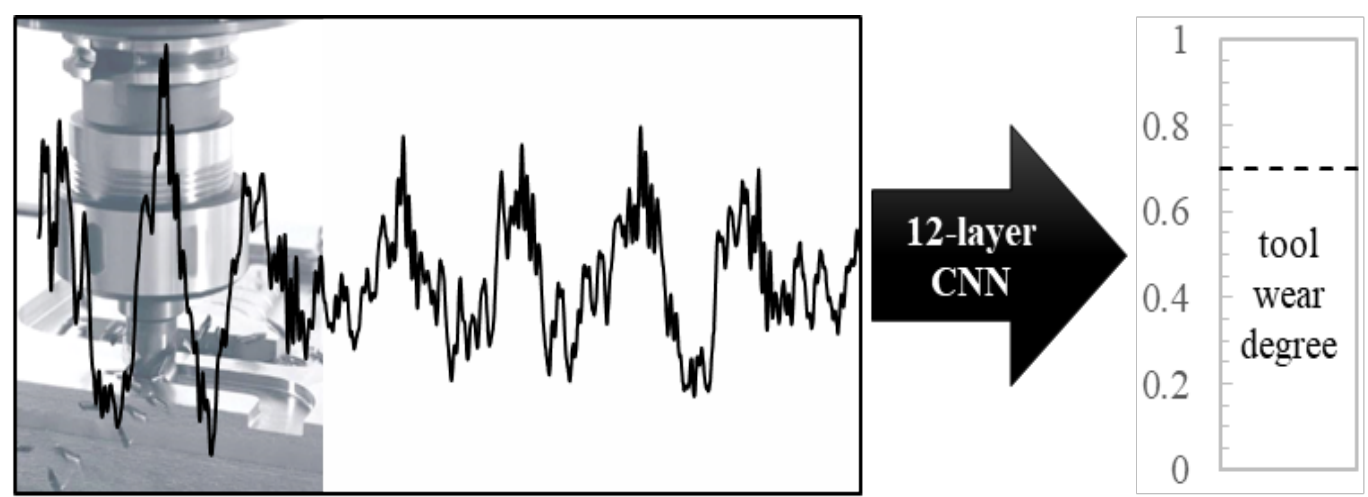

Fig. 1 Our trained convolutional neural network correctly predicts the wear of milling cutters from this cutting forces signal recorded with a Kistler quartz 3-component platform dynamometer

Fig 2 shows a waveform diagram of the $\mathrm{X}$ direction force signal corresponding to the different wear stages. The whole data set contains 64000 sets of such data. The horizontal axis in the picture indicates time. The actual sampling frequency of the signal is $50 \mathrm{KHz}$. The longitudinal axis represents the $\mathrm{X}$ direction force signal, and the unit is $\mathrm{N}$. The title of picture indicates the degree of wear, and its value is between 0 and 1 , the larger the value, the more serious the tool wear is. [0.1554815]
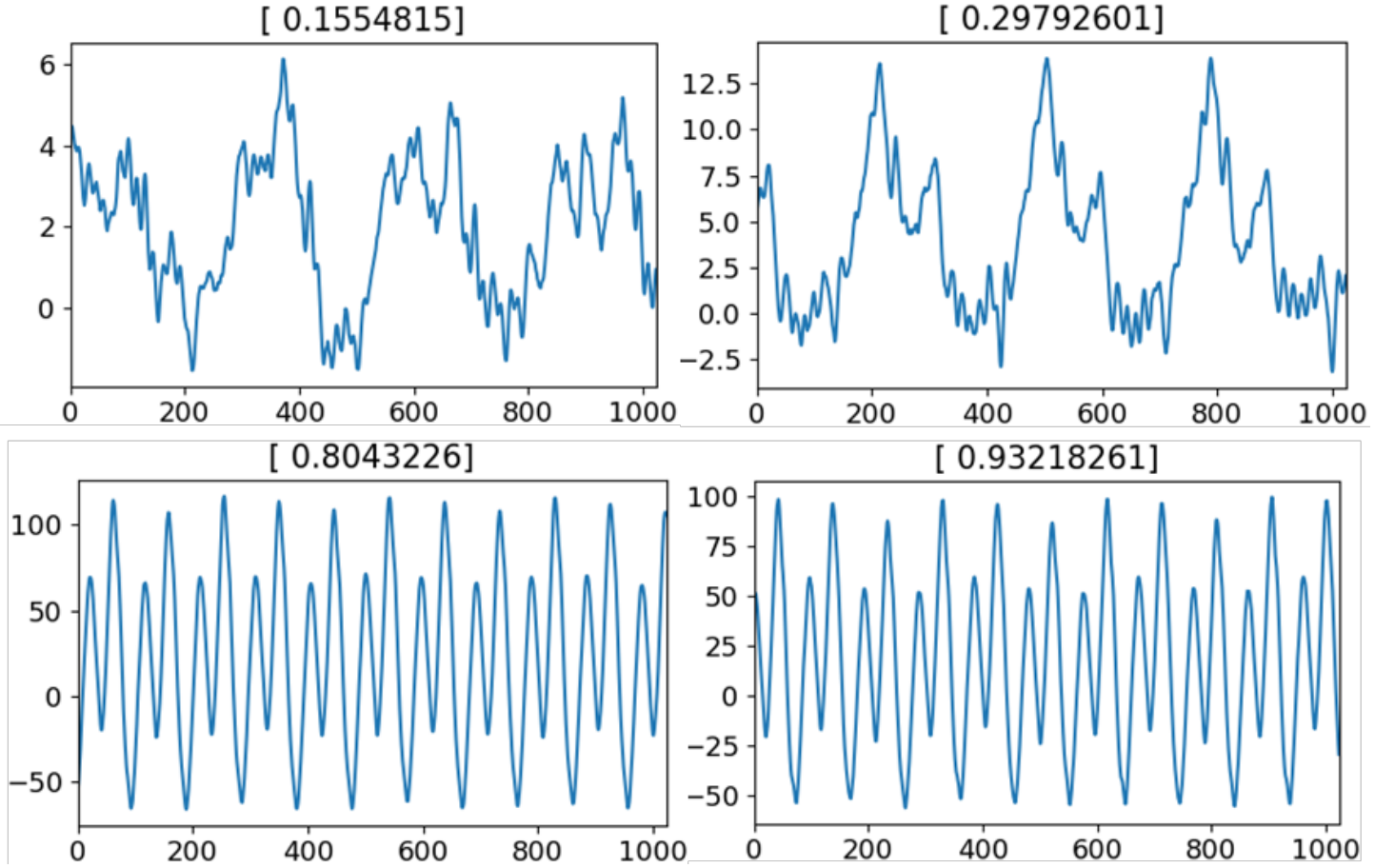

Fig. 2 Time varying $X$ direction force signals corresponding to cutting tools at different wear stages

It can be seen from the fig 2 that with increasing of tool wear, the cutting force signal changes not only in amplitude and frequency, but also in the waveform of signal. In the early stage of tool wear, the fluctuation of force signal is very intense. With the aggravation of wear, the waveform becomes very regular and its periodicity is more obvious. The method of predicting tool wear based on the fine features of the waveform is difficult to achieve in the traditional signal processing. With the continuous development of neural network algorithms, the idea of recognition through the subtle features of the image is realized in the convolution neural network. Therefore, in this study, we hope to build a prediction model based on convolutional neural network for cutting force signal and tool wear process, which is not only a more precise algorithm for tool wear prediction, but also a new direction for the application of convolution neural network. 


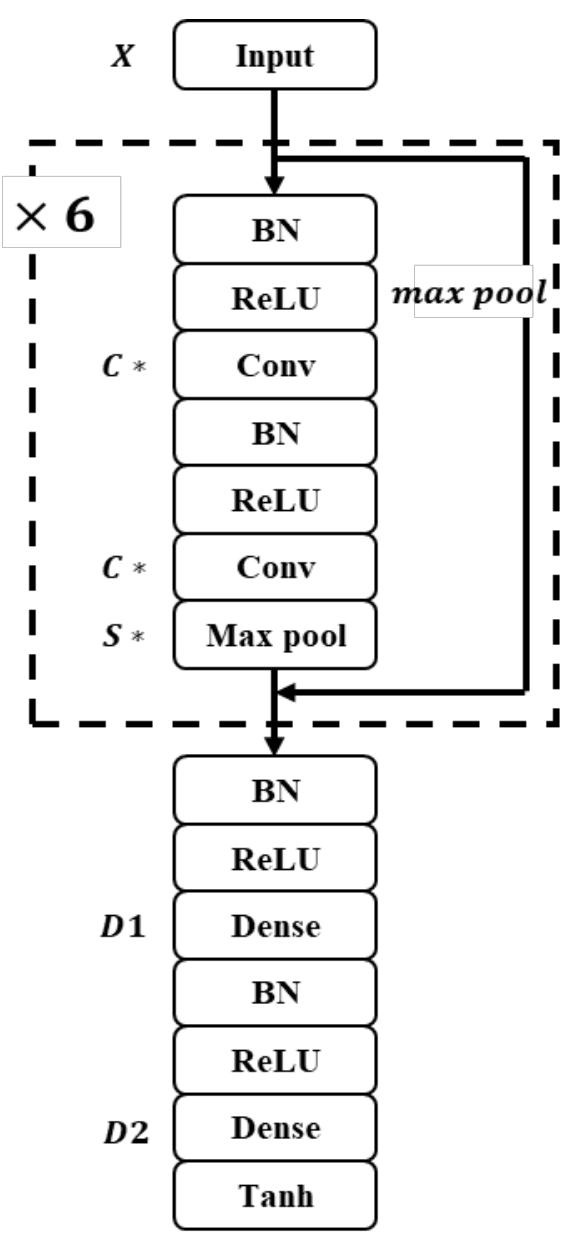

Fig. 3 The architecture of the network

\section{The Structure of the Model}

The structure of the convolution neural network, as shown in Fig 3, contains 7 coiling layers, 4 pool layers, 2 dense layers, and 8 Batch Normalization layers. The model structure used in this study adopts the idea of residual network design, and improves the design based on the model used by Wu [8] in the arrhythmia detection of ECG. The input X, as shown in Fig 3, is a vector with a length of 1024. Each convolution layer in the model contains 32 convolution kernel with a length of 8 . The matrix after convolution is consistent with the size of the pre convolution matrix. Since convolution layer is connected to Batch Normalization layer, there is no need to add bias after convolution layer.

In the model, "ReLU" means that the rectified linear units is used for nonlinear transformation of the characteristic matrix. The "dense" represents the dense layer. The output part of the convolution neural network has two dense layers, also known as the fully connected layer, with 256 and 1 neurons respectively. The network ends up with a "tanh" activation function that limits the output of the network between -1 and 1 . We apply Batch Normalization Before each convolutional layer to prevent the model from falling into over fitting and to accelerate the convergence of our model. We also apply dropout between the two full connected layers to prevent the model from getting into over fitting.

\section{Training and Testing}

In the model, the mean square error is set as the loss function.

$$
\mathrm{L}\left(\mathrm{X}, \mathrm{s}^{*}\right)=\frac{1}{\mathrm{n}} \sum_{\mathrm{i}=1}^{\mathrm{n}}\left(\mathrm{s}_{\mathrm{i}}-\mathrm{s}_{\mathrm{i}}^{*}\right)^{2}
$$

where " $\mathrm{s}_{\mathrm{i}}$ " is the output of the model when the input is $\mathrm{x}$, and " $\mathrm{s}_{\mathrm{i}}{ }^{*}$ " is the actual tool wear degree corresponding to the input " $\mathrm{x}$ ". Before training the model, all the data were randomly divided into 
training set and test set, in which the training data accounted for $90 \%$. The test set will only be available while testing the model but will not be used while training the model. The Adam optimizer was used with default settings and the learning rate was reduced by a factor of 2 if the testing loss stops improving. Finally, the best model was selected and saved according the evaluation result on the testing data set during training process.
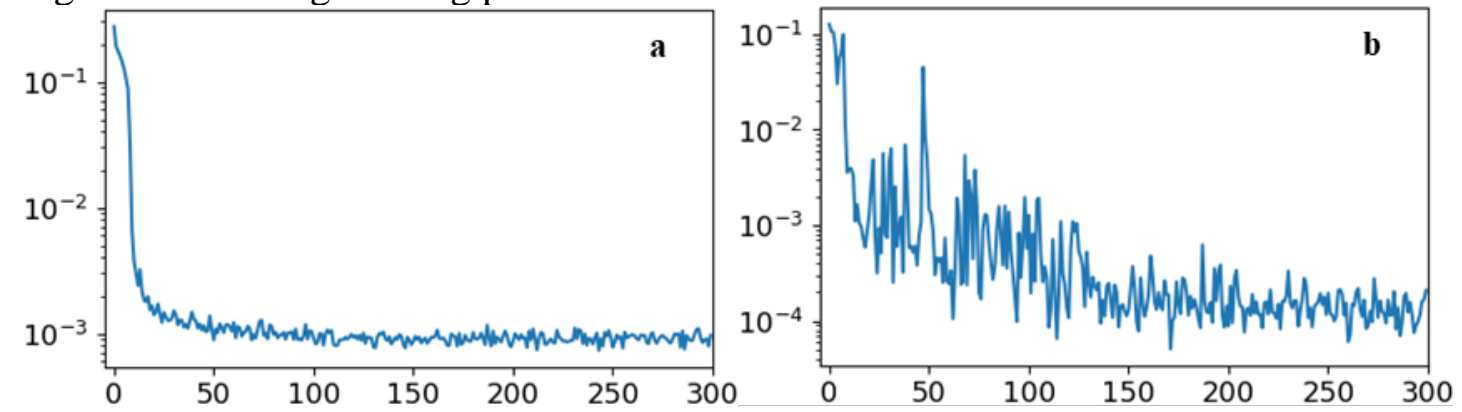

Fig. 4 The error of the model in the training set (a) and testing set (b) varies with the training times

Fig 4 shows the error of the model in the training set and test set varies with the training times. The figure is drawn using a semi logarithmic coordinate system.

Then regression analysis is made in order to study the prediction precision of the model. Fig 5 shows the prediction results of the model on testing set compared with the actual tool wear degree. The mean square error of the model on the testing set is $6.1 * 10^{-4}$.

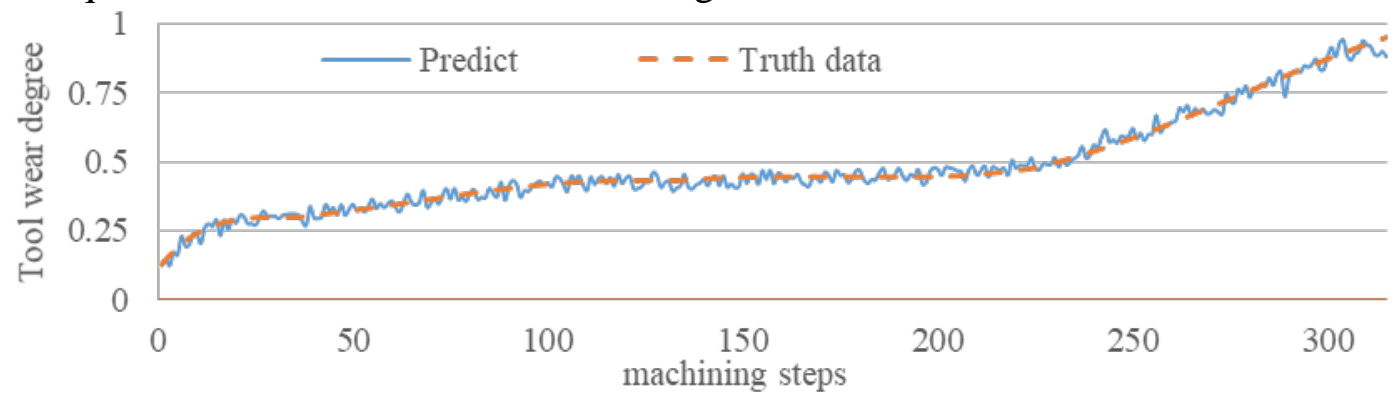

Fig. 5 Regression analysis of the convolution neural network model

\section{Summary}

In this work, the convolution neural network algorithm with deep residual network has been successfully used to solve the tool wear prediction problem. It maps a force signal fragment with a length of 1024 to a value in range $(0,1)$, and achieves excellent prediction results. However, in the data set we collected, all processing parameters and processing materials remain unchanged during data acquisition, so we could not know the prediction effect of the model under different processing parameters.

In addition, we plan to carry out more data acquisition experiments, which can be applied to tool wear prediction as early as possible, based on high precision force signal sensors. Finally, the tool state monitoring technology will be used to reduce the processing cost and to improve the machining quality during the machining process.

\section{References}

[1] Lécun Y, Bottou L, Bengio Y, Gradient-based learning applied to document recognition, Proceedings of the IEEE, (1998), 86(11):2278-2324.

[2] Krizhevsky A, Sutskever I, Hinton G E, ImageNet classification with deep convolutional neural networks, Communications of the Acm, (2012), 60(2):2012. 
[3] Simonyan K, Zisserman A, Very Deep Convolutional Networks for Large-Scale Image Recognition, Computer Science, (2014).

[4] Szegedy C, Liu W, Jia Y, et al, Going Deeper with Convolutions, (2014):1-9.

[5] He K, Zhang X, Ren S, et al, Deep Residual Learning for Image Recognition, (2015):770-778.

[6] Li X, Lim B S, Zhou J H, Fuzzy neural network modelling for tool wear estimation in dry milling operation, (2009).

[7] Čuš-Uroš F, Župerl, Real-Time Cutting Tool Condition Monitoring in Milling, Strojniski Vestnik, (2011), 57(2).

[8] Rajpurkar P, Hannun A Y, Haghpanahi M, Cardiologist-Level Arrhythmia Detection with Convolutional Neural Networks, (2017). 